\title{
La educación en América Latina: desarrollo y perspectivas
}

Education in Latin America: development and perspectives

\author{
Volumen 17, Número 2 \\ Mayo- Agosto \\ pp. 1-23
}

\section{Elmys Escribano Hervis}

Revista indizada en $\underline{\text { REDALYC, }}$ SCIELO

Revista distribuida en las bases de datos:

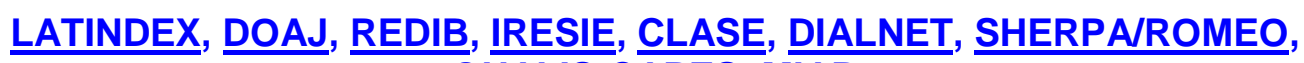
QUALIS-CAPES, MIAR

Revista registrada en los directorios:

ULRICH'S, $\underline{\text { REDIE}}, \underline{\text { RINACE}}, \underline{\text { OEI }}, \underline{\text { MAESTROTECA, PREAL, }}$ 


\title{
La educación en América Latina: desarrollo y perspectivas
}

\author{
Education in Latin America: development and perspectives
}

\section{Elmys Escribano Hervis ${ }^{1}$}

Resumen: El tema de este ensayo es la manifestación actual de la educación, su desarrollo y sus perspectivas en América Latina. El propósito esencial es valorar la educación, su estado actual y sus principales desafíos. Se aplica el método comparativo que correlaciona e integra un conjunto de variables e indicadores que se expresan de manera particular en el continente, en cada región o país. Se señala la pobreza y la exclusión como variable contextual determinante en la educación. Este factor aparece unido a otros elementos históricos, culturales y políticos. Como resultado de la valoración, se reconoce los avances en materia educativa en el continente, así como sus principales demandas y perspectivas de desarrollo manifiestas en: la inversión en la educación, la evaluación de la calidad de la educación, las políticas relacionadas con las TIC y la necesidad de subrayar la importancia del docente en la sociedad actual.

Palabras clave: América Latina, pobreza, modelo educativo, evaluación de la educación, calidad de la educación, TIC.

\begin{abstract}
The theme of this essay is the current manifestation of education and its development prospects in Latin America. The essential purpose is to value education, its status and its main challenges. The comparative method that correlates and integrates a set of variables and indicators that are expressed particularly on the continent, in each country or region is applied. Poverty and exclusion as a determining contextual variable in education noted. This factor appears together with other historical, cultural and political elements. investment in education, quality evaluation of education policies related to the: As a result of the evaluation, advances in education on the continent, as well as their main demands and perspectives of gross development is recognized ICT and the need to stress the importance of teaching in today's society.
\end{abstract}

Keywords: Latin America, poverty, educational model, educational evaluation, educational quality, ICT.

\footnotetext{
1 Profesor titular de la Universidad de Matanzas, Cuba. Doctor en Ciencias Pedagógicas del Instituto Central de Ciencias Pedagógicas, Cuba. Director de la Revista ATENAS.
}

Dirección electrónica: escriba2003@gmail.com

Ensayo recibido: 23 de junio, 2016

Enviado a corrección: 5 de octubre, 2016

Aprobado: 13 de febrero, 2017 


\section{Introducción}

Los estudios comparatistas de la educación son de gran utilidad para distintas esferas de la vida pública. La ciencia debe ofrecer métodos y resultados, así como diagnóstico y pronóstico del objeto que es necesario atender y transformar. Este tipo de análisis es pertinente y de gran provecho en la actualidad, pues además de brindar una información que pudiera resultar más o menos relevante para estudiantes y profesionales de la educación, es de utilidad para decisores de políticas públicas en relación con la educación en todas sus aristas.

A pesar de la gran diversidad cultural que impera hoy en América Latina, se intenta una generalización en el tema analizado. Se asume la educación como un fenómeno social complejo y dialéctico. Para su análisis, es primordialmente necesario contextualizarla a nivel de región, subregión y país, e incluso dentro de cada país se concreta de manera diferente en correspondencia con los factores condicionantes, pues la educación aparece sometida a la influencia de los factores económicos, tanto por sus ritmos de crecimiento, como por sus limitaciones. Es necesario considerar factores como la pobreza y la exclusión, la distribución de la riqueza y correlacionado con ello el gasto o las inversiones y las implicaciones manifestadas en la educación, por solo mencionar algunos elementos condicionantes.

De igual forma, se señala la significación de la historia y la cultura como referentes esenciales para la educación y la escuela actual en América Latina.

El propósito de este artículo es describir el estado actual de la educación en América Latina y, sobre la base de la correlación con algunas variables que se señalan, establecer algunos puntos que se consideran esenciales para su desarrollo perspectivo. Para la realización de este estudio, se han sometido a análisis, informes publicados por organizaciones internacionales, así como trabajos académicos de significación en el tema que ofrecen valoraciones y datos, los cuales son referidos y analizados dentro de una lógica compleja e integral que contextualiza la educación en un área marcada significativamente por la diversidad. Por la naturaleza y proyección de este trabajo, se aplica el método comparativo que correlaciona e integra un conjunto de variables e indicadores expresados de manera particular en cada país o región.

\section{Planteamiento del tema y algunas precisiones metodológicas}

La educación en Latinoamérica como objeto de estudio es un fenómeno complejo. Resulta arriesgado y difícil establecer generalizaciones lo suficientemente abarcadoras del 
mosaico de situaciones que representa la región y cada país. Uno de los rasgos más destacados del territorio es su diversidad; se aprecian desigualdades notables entre unos países y otros, e incluso, en el interior de cada uno de ellos (Organización de Estados Iberoamericanos para la Educación, la Ciencia y la Cultura - OEI, 2014).

La educación como expresión de las relaciones sociales está condicionada, ante todo, por las circunstancias económicas y políticas. También está influenciada por factores culturales específicos de cada nación, como puede ser la historia, y las tendencias de desarrollo actual. "Con el fin de valorar de manera adecuada la respuesta que los sistemas educativos son capaces de ofrecer, es necesario situarlos en sus respectivos contextos" (OEI, 2014, p. 36).

En la variable de contexto uno de los factores más relevantes por tomar en cuenta en estudios sobre la educación en la región latinoamericana es la condición de pobreza de las familias. La situación de pobreza limita el acceso a la educación y obstaculiza el aprovechamiento del estudio y de las oportunidades. Es necesario apreciar de modo dinámico y multidimensional el problema de la pobreza y su incidencia en la educación en América Latina en relación con los diferentes indicadores que pueden ser considerados y a la combinación en que se manifiesten en cada contexto en la vida social (García, Caamal y Prieto, 2016; Pérez, 2003; García, 2014).

Fuentes de la Oficina Regional de Educación para América Latina y el Caribe (Orealc/Unesco), señalan que, entre 2000 y 2010, el promedio de personas cuyos ingresos les situaban bajo la línea de la pobreza disminuyó desde 43,9\% hasta 35,4 \%, entre los países del área. Los casos de Bolivia, Venezuela y Argentina se destacan particularmente por haber reducido prácticamente a la mitad, sus tasas de pobreza durante el período.

A pesar de estos avances, no se debe perder de vista que la incidencia de la pobreza en América Latina continúa siendo un enorme obstáculo para la expansión y mejoramiento de la educación de su población infantil y juvenil, al afectar en promedio a alrededor de un tercio de la población de cada país. (Orealc/Unesco), 2013, p. 17)

En el contexto descrito, la tasa de pobreza de América Latina en 2013 fue de un 28,1\% de la población, en tanto que la indigencia, o pobreza extrema, alcanzó el 11,7\%. Estos porcentajes equivalen a 165 millones de personas en situación de pobreza, de los cuales 69 millones son personas en situación de pobreza extrema. Estos valores dan cuenta de que la tasa de pobreza se mantuvo sin grandes diferencias con respecto a los niveles observados en 2012 (28,1\%). La pobreza extrema, por su parte, mostró una 
leve tendencia al aumento, situándose 0,4 puntos porcentuales por encima del valor de 2012 (11,3\%). Esto significa que en 2013 el número de personas pobres se mantuvo sin cambios mayores, en tanto que el número de personas en situación de indigencia se incrementó alrededor de 3 millones. (Comisión Económica para América Latina y el Caribe, 2014, p. 64)

La expresión cuantitativa de grupos humanos en situación de pobreza extrema en el continente es significativa. En igual medida, se manifiesta las limitaciones y afectaciones a su modo de vida y, por consiguiente, en sus escasas posibilidades de acceso a la educación como base de su desarrollo humano.

Es esencial correlacionar la riqueza generada y cómo esta se distribuye, al tiempo que esta condición satisface, en mayor o menor medida, las necesidades de bienestar y formación de una sociedad en un momento histórico dado.

América Latina y el Caribe es la región con el mayor contraste y desequilibrios en la distribución del ingreso en el mundo. Por tanto, aunque el PIB per cápita proporciona una buena imagen de conjunto de la situación económica de los países, la existencia de fuertes desigualdades en América Latina al interior de los países obliga a tomar en cuenta la forma en que dicho crecimiento se distribuye entre los hogares. En este sentido, el avance ha sido muy lento en la última década, puesto que el coeficiente Gini $^{2}$ de distribución del ingreso apenas se redujo de aproximadamente 0,53 a 0,51 entre 2000 y 2010, manteniéndose en niveles comparativamente muy elevados. (Orealc/Unesco), 2013, p. 16)

Muy relacionado con el análisis de la relación pobreza-educación se encuentra la presencia de población originaria en la región. Este tipo de población se concentra básicamente en zonas rurales, lo que representa dificultades adicionales a la expansión de la educación formal en América Latina y el Caribe, para poder satisfacer las necesidades de acceso con equidad y posibilidades reales de desarrollo para todos. Se le adiciona a la problemática, barreras en la comunicación y el acceso a dichas áreas poblacionales. En efecto, como promedio, en los países de la región, alrededor de 4 de cada 10 personas vivían en zonas rurales en 2010. Por un lado, la Orealc/Unesco (2013), expone: "Más aun,

${ }^{2}$ El coeficiente de Gini es un parámetro que mide el nivel de desigualdad en la distribución del ingreso en una escala que va de 0 a 1 , dependiendo del grado de concentración. De esta manera, un coeficiente de 0 sería indicativo de la inexistencia de desigualdad, en tanto que un parámetro de 1 significaría una total concentración de la riqueza 
en 2010, en 12 de los 35 países para los que se cuenta con información, la mitad o más de su población vivía en zonas rurales". Por otro lado, la Organización de Estados Iberoamericanos para la Educación, la Ciencia y la Cultura (OEI), manifiesta: "Junto con el nivel socioeconómico y cultural, en algunos países pertenecer a una población originaria o ser afrodescendiente puede ser también un factor de vulnerabilidad" (2014, p. 93).

Los estudios efectuados con finalidades analíticas y comparativas en la educación por regiones, además de la variable económica con los indicadores necesarios, localizan otras variables importantes como la demográfica; en tanto es necesario establecer la densidad poblacional en diferentes áreas, por ejemplo, la densidad poblacional en zonas urbanas, en zonas rurales, las tendencias de movilidad y cambio entre estas, así como la significación de la población económicamente activa. Hay que tomar en cuenta también la urbanización de la población y la prevalencia de acuerdo con los rangos de edades. En 2012, la estimación del Banco Mundial "es de 644 millones de habitantes en Iberoamérica, 14 millones más que en 2010 y 88 más que en 2000 (un 15,8 \% más de habitantes en 2012 que en 2000, y un 2,1\% más que en 2010" (OEI, 2014, p. 37).

Como se aprecia, el estudio de la educación en el continente requiere tomar en cuenta, en su justa medida, las variables asociadas a la economía, las políticas educativas, los factores demográficos y, sobre todo, los elementos internos que se asocian a la estructura y el dinamismo de los sistemas educativos, así como las tendencias de desarrollo, que en la época actual suelen asociarse con las políticas relacionadas con las TIC y su empleo en la educación.

Debe tomarse en cuenta la capacidad manifiesta de cada sistema educativo para concretar la esperanza de vida escolar -es decir, la cantidad de años probables que pueda permanecer en el sistema educativo un niño incorporado a él-, debe también observarse el nivel formativo alcanzado por la población adulta, el número de días lectivos al año, las estadísticas del alumnado y profesorado y el resto del personal no docente.

Como se expresó al inicio, cada uno de estos aspectos influye de una manera particular en la educación y la escuela, muchas veces expresando una mezcla muy singular entre cada uno de los factores en correspondencia con el contexto y el momento histórico en que se analice.

La insatisfacción de las necesidades en materia de educación en América Latina es tan larga como su propia historia y expresa las inequidades sociales y carencias que se manifiestan en la región. Los modelos educativos adoptados en cada época de la historia en el continente se hacen corresponder con la sociedad, su cultura y el entramado de relaciones 
impuesto por la política y la economía para la región y cada país en particular (Escribano, 2015; Weinberg, 1981).

\section{Las lecciones de la historia de la educación en América Latina}

Para tener una percepción objetiva del estado actual de la educación en América Latina y determinar sus tendencias de desarrollo, se hace imprescindible determinar los rasgos esenciales de la educación en el pasado. La visión más generalizada con que se ha asumido la educación ha estado en sintonía con el ideal iluminista del siglo XVIII. Según Torres (2001): "La expansión y universalización de la educación aparece, así como un signo de la modernidad, un signo de mayor racionalidad de la acción social, pero paradójicamente, también como una precondición para que éstas se den" (p. 24).

El arribo de América Latina a la llamada modernidad, como proceso histórico, se hacía corresponder con sus necesidades de desarrollo y libertad más perentorias, pero a la vez tomaba como referencia la experiencia de Europa, especialmente Francia y Estados Unidos. Los líderes que encabezaron la gesta independentista en el continente fueron hombres ilustrados, se aprecia con facilidad que sus ideas por momentos se presentan como continuidad de un régimen $\mathrm{y}$, al mismo tiempo, como ruptura con este. Sus discursos transidos de la idea de la libertad manifiestan sus intereses de clases que en aquella compleja situación histórica coincidían con la fundación de nuestras naciones. Para encarar un reto de tal magnitud, estos hombres depositaron toda su confianza en la educación y la cultura. Identificaron la educación popular como consustancial al proceso revolucionario independentista y le adjudicaron una potencialidad transformadora desmedida (Escribano, 2016).

La práctica demostró que la vida y los profundos problemas sociales arraigados en las sociedades de entonces no se solucionaban con legislaciones cuyo cumplimiento, por demás, era dudoso y poco sistemático. Paz (1993) ha afirmado que en esta época "Hispanoamérica fue una España sin España (...) Un feudalismo disfrazado de liberalismo burgués, un absolutismo sin monarca pero con reyezuelos: los señores presidentes" (p. 126).

Los gobiernos establecidos en Latinoamérica en el período posterior a las guerras de independencia, aunque hicieron la revolución y tejieron la idea de la independencia con una base agraria, a la postre desconocieron la esencia humana de la base social con la que se hizo la independencia. En materia educacional, existía un escuálido sistema sustentado en decretos y leyes extranjerizantes y ajenas a la realidad y a las necesidades de bienestar, 
independencia y desarrollo de las sociedades naturales de estas tierras. En otros casos se mantenían obsoletos sistemas educativos en aquellas repúblicas, que su viejo cuerpo lastrado por la inconformidad con la época reclamaba cambios (Escribano, 2016).

En su corta estancia en Venezuela, en 1881, le fue suficiente a José Martí para expresar una aguda observación a esa sociedad y a las contradicciones que encaraba su cultura por aquella fecha. Escribió que los pueblos de América:

Estaban criados como parisienses, se ahogan en su país: no sabrían vivir bien más que en París. Son plantas exóticas en su propio suelo: lo cual es una desgracia (...) Cuando el pueblo en que se ha nacido no está al nivel de la época en que vive, es preciso ser a la vez el hombre de su época y el de su pueblo, pero hay que ser ante todo el hombre de su pueblo. (Martí, 1963, Vol. 7, p. 154)

La imitación y el desarraigo que observó en la sociedad caraqueña de entonces le permitió a Martí reflexionar sobre su significación para estos pueblos, sus habitantes y la necesidad de fraguar la verdadera independencia en las ideas, la educación y la cultura.

En la ciudad, una vida rara semipatriarcal, semiparisiense, espera a los forasteros. Las comidas que en ella se sirven, exceptuando algunos platos del país, las sillas para sentarse, los trajes que se usan, los libros que se leen, todo es europeo (...) se desdeña el estudio de las cuestiones esenciales de la patria; -se sueña con soluciones extranjeras para problemas originales;- se quiere aplicar sentimientos absolutamente genuinos, fórmulas políticas y económicas nacidas de elementos completamente diferentes. (Martí, 1963, Vol. 19, pp. 159-160)

Además de las incongruencias y asimetrías en la educación y la cultura, comentadas aquí brevemente, hay que señalar que la economía latinoamericana mantuvo esencialmente su carácter agrario sin que aparecieran proyectos propios de desarrollo capaces de desestancar las naciones.

El siglo XIX cerró con un aliento político liberal que tuvo pronta repercusión en la educación y en la cultura. La necesidad de orden y progreso para estas naciones representó un terreno abonado para la entrada del positivismo. Esta postura filosófica no fue asumida de forma mecánica, ni totalmente acrítica, sino que se tomó en cada país como fundamento de un necesario proceso de cambio en la educación, depositó confianza en el ser humano, en la ciencia, optó por el desarrollo de la industria, lo cual era sin dudas un avance si se contrasta 
con las concepciones de las oligarquías retrógradas y burocráticas instaladas en el poder (Guadarrama, 2008a).

A pesar del entusiasmo positivista en la etapa, el Estado Liberal no resolvió el problema de la educación, no fue superada la diferencia entre la educación que se dispensaba y las necesidades básicas de los pueblos. La solución del problema de la educación de las poblaciones del campo continuó siendo postergada. En 1900, el 54 \% de los habitantes de nuestra América eran analfabetos. El vínculo orgánico entre proyecto educativo, proyecto social de transformación del ser humano, continuaba ausente. Las políticas de desarrollo económico eran débiles e inestables, cuando no comprometidas con los intereses de alguna lejana potencia extranjera (Escribano, 2016).

Más tarde, el denominado Estado Desarrollista apostó a la educación como una inversión en recursos humanos, las estadísticas muestran mejoras considerables en el acceso, pero no pudieron ser erradicadas las inequidades y problemáticas que ya se convertían crónicas en la educación en el continente. El Estado Neoliberal marcó la tendencia de privatización presente en todos los niveles educativos y la descentralización en el control de la educación.

Especialmente hacia el fin del siglo, [el autor citado se refiere al fin del siglo $X X$ ] los estados latinoamericanos, prisioneros de las contradicciones del desarrollo latinoamericano y de sus propias lógicas del actuar burocrático, todavía relamiéndose las heridas de la doble crisis de la deuda externa y el déficit fiscal que se arrastra desde los ochenta como un yugo aparentemente imposible de superar, encuentran cada vez más dificultades para atacar los problemas existentes en el mundo de la educación, fundamentalmente por las dificultades del financiamiento educativo, (...) también en función de filosofías políticas y agendas educativas que postulan una privatización de la educación y el abandono de las responsabilidades estatales en la educación pública. La educación elemental y secundaria de la región continúa siendo segregada por clases sociales: los pobres estudian en la escuela pública, y los sectores medios y altos florecen educativamente en instituciones privadas. (Torres, 2001, p. 24)

La falta de control estatal en la educación se vinculó también con su carencia de responsabilidad con el financiamiento y la sustentación de políticas educativas articuladas en respuesta a las necesidades de desarrollo fieles a nuestra cultura mestiza. 
En esta región ha pesado sobre la educación las estrecheces financieras que ha impedido la creación de la infraestructura necesaria, cubrir las demandas de acceso y el estímulo verdadero de las apuestas por el desarrollo; además, la persistencia del analfabetismo en todas sus expresiones (el analfabetismo clásico, pudiéramos decir, el analfabetismo funcional y por último el llamado analfabetismo digital, que se localiza en la cultura o la sociedad de la información), este lastre social se hace una carga especialmente para las mujeres y los sectores más humildes que residen en áreas rurales, incluida la población originaria. No podría faltar en esta mención de factores que ponen en riesgo la educación en esta región, los socorridos "préstamos intelectuales" del extranjero para la conformación de sistemas educativos nacionales, el diseño de políticas educativas o la realización de reformas que traicionan, como ya se dijo, la cultura y la historia, tan rica, original, auténtica y diversa.

\section{Un acercamiento al estado actual de la educación en América Latina}

Existe coincidencia entre los autores que abordan el tema en señalar que, en las últimas cinco décadas del siglo XX, la educación en América Latina experimentó mejoras significativas (Torres, 2001).

Un indicador de los cambios se observa en el acceso. La expansión de los servicios educacionales estuvo asociada al despegue industrial que operó el continente sobre todo en las décadas del 50 y el 60 del referido siglo. "Entre 1960 y 1970, los índices de crecimiento de la educación superior y la educación media fueron $247,9 \%$ y $258,3 \%$, respectivamente" (Unesco, 1974, p. 167). Sin embargo, señala Carlos Alberto Torres (2001) que el crecimiento en el acceso a la educación primaria, aunque resultó significativo (167,6\%), no se hacía corresponder con el aumento en los otros dos niveles. La diferencia pudiera resultar comprensible si se parte de considerar las estructuras poco coherentes de los sistemas educativos y las debilidades en el acceso concentradas básicamente en esos niveles. Se denota, además, que las tasas de alfabetización se mantuvieron relativamente constantes en la mayoría de los países de la región latinoamericana.

El gasto en función de la educación no ha sido estable ni se ha comportado de manera eficiente; tampoco ha sido proporcional al aumento del PIB; aunque se aprecie un crecimiento de la inversión en educación, no lo hace al ritmo que se necesita.

En materia de reformas se han acumulado críticas e insatisfacciones que apuntan a una falta de legitimidad de los sistemas educativos, agravados por males como la deserción 
escolar, la repetición o la relevancia de la formación entendida como preparación efectiva de los educandos para la vida. A esta situación se suma la incoherencia entre las influencias de los profesionales de la educación, la familia y las instituciones (Torres, 2001).

El escenario educativo en la contemporaneidad se ha visto influido por el fenómeno TIC, lo cual ha contribuido a señalar expectativas y dinamizar la educación, o al menos a percibirla de manera potencial fuera de los límites físicos de la escuela y de manera efectiva como proceso a lo largo de toda la vida.

La primera política a favor de la integración de las TIC al desarrollo se plasmó en los objetivos de desarrollo del milenio. A fines de la década de los años 80 y principios de los años 90, se comenzaron a gestar las primeras políticas y programas TIC orientados a las escuelas (Instituto de Estadística de la Unesco, 2013). Para el caso de América Latina, en primera instancia, la aparición del fenómeno TIC en educación dependió de la política pública y de la aparición de programas y proyectos, los cuales estimularan el empleo de las TIC en las escuelas, y esto ha despertado grandes expectativas, pues esta meta estuvo asociada a un ideal de calidad y eficiencia, unido a la equidad, que la realidad se ha encargado de señalar en cuanto a la diferencia entre lo logrado y lo esperado. Los resultados han sido modestos en el mejor de los casos. Cada aspecto por sí mismo, la idea de la calidad y la eficiencia de la educación mediada por TIC, requiere condiciones tecnológicas y recursos humanos bien preparados.

En el segundo caso, las TIC, como herramienta para garantizar equidad, son también un caso difícil.

Hasta ahora la brecha digital en América Latina ha sido concebida en términos de acceso a la tecnología y, desde ese punto de vista, la escuela ha sido pensada como un espacio estratégico para reducir las desigualdades de acceso. Sin embargo, investigaciones recientes comienzan a hablar de la emergencia de una segunda brecha digital. Esta se refiere a la necesidad de considerar no sólo las diferencias en términos de acceso a las TIC y el desarrollo de destrezas de manejo funcional de las mismas, sino también en términos de las capacidades de los estudiantes de diferente contexto sociocultural de dar un uso efectivo de las tecnologías para su aprendizaje. Esta segunda brecha digital distingue entre aquellos que tienen las competencias y habilidades necesarias para beneficiarse del uso de los computadores y quienes no las tienen, competencias que están estrechamente vinculadas al capital social, económico y cultural de los estudiantes. (Sunkel, 2011, párr. 9) 
Asimismo, según el Instituto de Estadística de la Unesco (2013): "En América Latina y el Caribe, 31 de 38 países (82\%) han adoptado, por lo menos, una definición formal respecto de iniciativas que utilizan las TIC en educación, mientras que en 9 países (24\%) todas son de carácter formal" (p. 7).

La misma fuente citada sostiene que la mayoría de los países hace alguna recomendación sobre la conveniencia de integrar la enseñanza asistida por las TIC a todos los niveles (educación primaria, primer y segundo ciclo de secundaria). "Quince países reportaron que sus programas de estudio incluyen recomendaciones sobre enseñanza asistida por TIC para todos los grados y asignaturas impartidas en primaria, y primer y segundo ciclo de secundaria" (Instituto de Estadística de la Unesco, 2013, p. 9).

En la región existen contrastes que van desde Uruguay, donde, a cada estudiante y a cada educador del nivel primario, el Estado le garantiza una computadora de modo gratuito. Por su parte, en el vecino Paraguay, 130 niños y niñas comparten una PC en la escuela del mismo nivel.

Todos los países tienen la responsabilidad de ofrecer educación para todos, garantizar el acceso con equidad y calidad en los servicios de cada institución educativa y el desempeño de los docentes. En tal sentido, la Unesco ha concebido sistemas internacionales de evaluación de la calidad de la educación.

El objetivo del TERCE NO es el ranking de los países, no busca detectar ganadores o perdedores en la región. El aporte del TERCE consiste en mostrar el avance de los países y los factores que explican su éxito o su rezago. Lo central no es "quién va ganando la carrera", sino entender cuáles son las razones de su éxito y, así generar aprendizajes mutuos entre sistemas educativos. Por tanto, más que comparar entre países, el análisis debiese ser a nivel país, ya que, si bien existen elementos comunes, la región presenta realidades muy diversas. (Unesco, 2014, p. 54)

Los resultados generales del $\mathrm{TERCE}^{3}$ en comparación con el $\mathrm{SERCE}^{4}$ fueron superiores. Se apreció una disminución de la proporción de estudiantes que se ubican en los

${ }^{3}$ El Tercer Estudio Regional Comparativo y Explicativo (TERCE) es un estudio sobre la adquisición de aprendizajes a gran escala. Se considera el estudio, dentro de los de su tipo, más importante de la región, ya que comprende 15 países (Argentina, Brasil, Chile, Colombia, Costa Rica, Ecuador, Guatemala, Honduras, México, Nicaragua, Panamá, Paraguay, Perú, República Dominicana y Uruguay) más el Estado de Nuevo León (México). El estudio se aplicó en el año 2013, evaluó el desempeño escolar en tercer y sexto grado de escuela primaria en las áreas de matemática, lenguaje (lectura y escritura) y, para sexto grado, el área de ciencias naturales. Su objetivo principal es aportar información para el debate sobre la calidad de la educación en la región, así como orientar la toma de decisiones en políticas públicas educativas. 
niveles de desempeño más bajos, y un leve aumento en la proporción de aquellos que se ubican en los niveles más altos (III y IV); especialmente en el nivel III. Aun así, resulta preocupante que los niveles I y II tienden a concentrar más del $50 \%$ de los estudiantes de primaria en la región tanto en SERCE como en TERCE. En particular, matemática en tercer grado es el área en la cual el porcentaje de estudiantes en nivel IV tuvo un mayor aumento, pasando de $8 \%$ a $12 \%$. (Unesco, 2014, p. 55)

Si bien el sentido de estas evaluaciones no es medirse con otros países, los resultados deben ser una fuente inspiradora para propiciar los cambios y las mejoras necesarias al interior de nuestros sistemas educativos. La idea de lograr aprendizajes para toda la vida y lograr las adquisiciones con calidad es un reclamo básico para los países de la región. Por un lado, Sánchez (2015, párrs. 17-18) plantea:

Más allá de sus carencias, pusieron la atención en el hecho de que la masiva expansión del acceso a la educación en la región, una gran victoria en sí misma, no es suficiente sin una mejora equivalente en calidad.

Los legisladores ya no pueden ignorar la realidad de que incluso los países con mejor desempeño en América Latina, mucho menos los medios, están muy lejos del mundo desarrollado y muy lejos del lugar donde necesitan estar para competir en la economía global.

Por otro lado, la Orealc/Unesco- (2013) expone:

El logro académico de los alumnos de la región es preocupante en la mayoría de los países para los que se cuenta con información: en promedio, aproximadamente un tercio de los alumnos en primaria y casi la mitad en secundaria no parecen haber adquirido los aprendizajes básicos en lectura; y en matemáticas los resultados son incluso más insatisfactorios. Además, existe una aguda inequidad en contra de los alumnos más desfavorecidos, especialmente los más pobres, en cuanto al logro académico. (p.10)

\footnotetext{
${ }^{4}$ El SERCE son las siglas del Segundo Estudio Regional Comparativo y Explicativo, realizado en el 2006. Evaluó el desempeño de los estudiantes de tercero y sexto grados de educación primaria en América Latina y el Caribe en las áreas de matemática, lenguaje (lectura y escritura) y ciencias. Paralelamente, se intentó explicar dichos logros identificando las características de los estudiantes, de las aulas y de las escuelas que participaron en la experiencia en América Latina y el Caribe.
} 
Comentamos en el gráfico 1, los resultados y experiencias que emanan de la prueba PISA de la OCDE, de acuerdo con los resultados de 2012, en la que participaron menos de la mitad de los países de la región.

Gráfico 1. Porcentaje promedio de estudiantes en cada nivel de desempeño en PISA por región. Matemática (M), Lectura (L) y Ciencias (C), por región, 2012

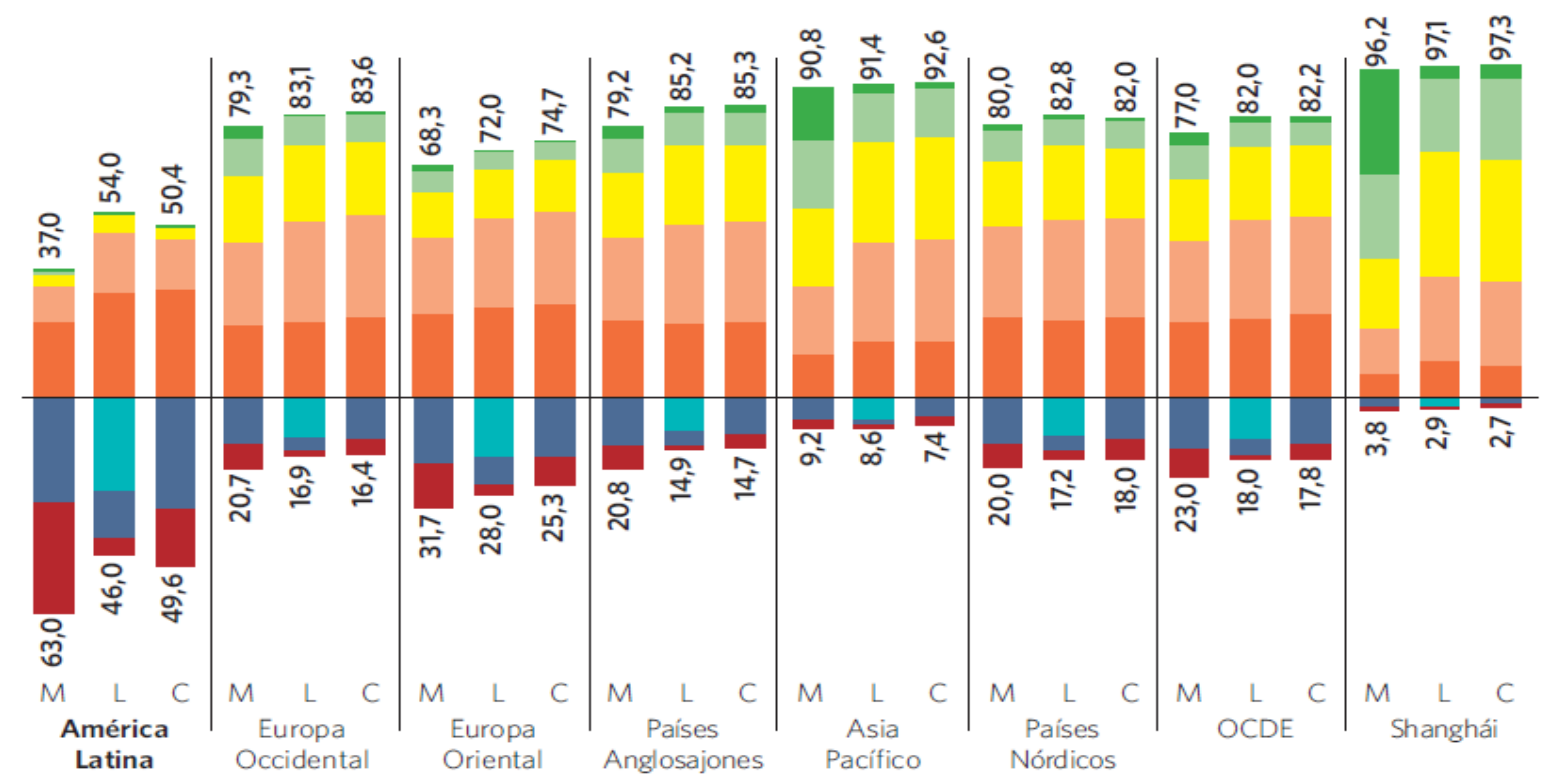

Debajo de nivel $1 \square$ Nivel $1 \square$ Nivel 1a (sólo para Lectura) $\square$ Nivel $2 \square$ Nivel $3 \square$ Nivel $4 \square$ Nivel $5 \square$ Nivel 6

Fuente: CIPPEC, sobre la base de OCDE, PISA. (2012) Citado por Rivas, A. (2015).

Como se aprecia, al comparar los resultados generales por regiones, para el caso de América Latina, concentra los índices más bajos. A pesar de las desconfianzas lógicas generadas por este tipo de pruebas y sus resultados, es necesario reflexionar: ¿Asumimos los modelos pedagógicos correctos para la región y cada nación? ¿Es eficiente la labor educativa de la escuela y con esta el desempeño de nuestros profesionales de la educación? ¿Resulta suficiente la inversión en la educación para el futuro de nuestras naciones?

El reconocido académico estadounidense Eric Hanushek (2015), profesor de la Universidad de Stanford, citado por BBC Mundo, señala que la educación obligatoria y universal es apenas el primer paso. Lo crucial es qué tipo de habilidades cognitivas se transmiten: "Los estudiantes de América Latina han estado aprendiendo mucho menos cada año escolar que sus pares en el este de Asia". Ya no basta saber leer y escribir, sino "tener la capacidad de comprender y usar con reflexión crítica la información, además de la 
capacidad de razonar con conceptos matemáticos y extraer conclusiones basadas en evidencia”. (Martins, 2015) ¿Por dónde empezar? Lo fundamental es llegar primero al nivel básico de matemáticas y ciencia como una base para un aprendizaje más profundo y una mejor habilidad de interactuar con otros a nivel de ideas y conceptos Los países con pocos recursos naturales deben hacer más énfasis en la educación de calidad y en formar el capital humano para promover el desarrollo, según el profesor citado: "El futuro de América Latina realmente depende de lo que pase en sus colegios" (Martins, 2015).

\section{Perspectivas y desafíos}

Tomando en cuenta las lecciones de la historia en la región de América Latina, de acuerdo con el estado actual de la educación y sus tendencias y perspectivas de desarrollo, es preciso identificar los desafíos que debe encarar la educación, la escuela, los agentes que intervienen en el proceso educativo y los decisores de políticas públicas.

En los salones de clases de hoy está fraguado el compromiso con el futuro más inmediato de nuestras naciones. Para países con economías que en ocasiones se muestran inestables, debe sopesarse muy bien cuánto se invierte y en qué se invierte. Esto es esencial para garantizar una movilidad sostenida hacia el desarrollo científico, económico y cultural de nuestros pueblos. En tal sentido, mencionaremos cinco aspectos que pueden identificarse como desafíos esenciales para la educación en América Latina.

\subsection{El modelo pedagógico justo para América Latina}

Enrumbar la educación de acuerdo con un modelo pedagógico pertinente y auténtico con fundamentos coherentes en correspondencia con la tradición, la cultura, la naturaleza y las demandas de los hombres y mujeres de estas tierras, es la principal necesidad del proceso de formación humana en América Latina.

No basta con inversiones solventes, ni con la última tecnología de la que se dispone; es preciso, primero, concebir qué tipo de personas necesita el continente, qué tipo de personas requiere cada país y cada región específica dentro de cada país. ¿Qué debe saber, cómo aprende y para qué aprende? Es tan importante la determinación de los contenidos por aprender, como la precisión de los valores, las cualidades y los sentimientos que ha de incorporar a su personalidad.

Se necesitan enfoques y modelos educativos contextualizados en la región, respaldados en líneas de investigación sólidas, que brinden la visión y los ejes claves 
para transformar la educación desde lo profundo, y superen el tradicionalismo que sigue vigente a pesar de las reformas. Este tradicionalismo se caracteriza por tener procesos curriculares por asignaturas, contenidos y evaluaciones basadas en pruebas, con énfasis en actividades formales y academicistas en el aula, dejando de lado lo afectivo, el proyecto ético de vida y el trabajo social, entre otros aspectos. (Tobón, 2015, p. 5)

Ser auténtico es un gran desafío ante la disyuntiva de despojarse de sí, en una carrera frenética de ser como otros, ignorando que los desafíos que enfrentamos y la historia no es la misma para todos. América Latina ha desarrollado una vertiginosa carrera a través de la historia, yendo de prisa a través de cada etapa, tan de prisa que no se ha aprendido lo suficiente de las experiencias vividas.

La problemática planteada no es de fácil solución. En determinadas coyunturas ha resultado más práctico buscar la solución fuera de nuestra geografía sobre la forma de gobierno, la orientación filosófica o el tipo de educación que es necesario adoptar. Sin embargo, en nuestra herencia pedagógica no ha faltado quien haya señalado la vía y el camino correcto. Las lecciones del Maestro Simón Rodríguez (1771-1854) son una magnífica expresión de originalidad para el continente (Rumazo, 2008), o aquel señalamiento sentencioso de José de la Luz y Caballero (1800-1862) que, en 1833, escribió: "Ni en la substancia ni en el modo debe concebirse un plan científico para La Habana como se concebiría para Londres o Berlín" (De la Luz, 1952, p. 241).

La orientación y sustentación teórica y metodológica que rigen los modelos y enfoques al uso es un tema básico. Es preciso lograr la profundidad y la coherencia en tales fundamentos, el ajuste a su tiempo y sobre todo a su sociedad, a su historia y a su cultura. Este es el desafío mayor. Algunos filósofos de nuestra región, entre los que se destacan el mexicano Leopoldo Zea (1976), el peruano Edgar Montiel (1995) o el cubano Pablo Guadarrama González (2008b) han brindado sólidos argumentos.

Es necesario erigir una verdadera filosofía de la educación que oriente el acto educativo y conceda coherencia a las ciencias de la educación y a las acciones e influencias que sean concebidas con esa finalidad. Una necesidad básica dentro de esta problemática se refiere a educar desde la identidad y para la identidad (Montiel, 1995).

Para tener un nuevo modelo educativo en Latinoamérica que aborde la diversidad en la unidad, se requieren enfoques o propuestas pedagógicas contextualizadas a los procesos socio-económicos y culturales de la región, como en su momento lo fue la 
pedagogía de Paulo Freire y el movimiento de la escuela nueva, con orientación a la generación de sociedades del conocimiento. Un ejemplo de ello es la socioformación, una propuesta que se viene construyendo de manera colaborativa entre docentes, expertos e investigadores de varios países latinoamericanos. Se centra en la formación integral en un contexto social, asumida como un derecho más que como un servicio, a partir de la realización de proyectos para resolver los problemas comunitarios, donde intervienen los docentes, los directivos, los pares, la sociedad y los padres mediante actividades conjuntas y articuladas. (Tobón, Gonzalez, Nambo y Vázquez, 2015)

\subsection{La visión de la calidad en la educación y su evaluación}

Queda claro que la expansión del acceso a la educación en la región es un gran logro, pero no es suficiente sin una mejora equivalente en calidad (Sánchez, 2015). Alcanzar las metas de universalización de la educación se corresponde con otros momentos históricos. La problemática actual es garantizar una educación de calidad, con aprendizajes sólidos y la adquisición de herramientas que permitan aprender a lo largo de toda la vida. Es prioritario avanzar en la determinación de un concepto de calidad de la educación y el trabajo sostenido de todos los actores del proceso en la gestión de la calidad.

(...) cada vez más los nuevos criterios con que se deben juzgar los avances en educación remiten a la calidad, más que a la simple expansión de la educación. La concepción de calidad que aplicamos es amplia e incluye no sólo logros, sino condiciones y procesos, no sólo aspectos académicos, sino sicosociales y ciudadanos. Éste es sin duda el aspecto en que la región se encuentra crónicamente retrasada. (Orealc/Unesco), 2013, p. 5)

Es necesario fomentar la investigación científica y la innovación en las instituciones educativas de todos los niveles de la región, como mecanismos fundamentales de gestar una educación de calidad en todos los espacios y por parte de todos los actores que intervienen en el proceso para el desarrollo y progreso de nuestras naciones. Parejo a ello, deben incrementarse los espacios de socialización y debate sobre el tema, así como incorporar los resultados y metodologías de evaluación que aplican organismos internacionales. Según Schwartzman (2001): "La investigación educacional moderna, y de alta calidad no es particularmente cara, y puede desempeñar un rol muy importante en el 
desarrollo regional de la educación, planteando temas, cuestionando políticas de dudosa efectividad y ayudando a señalar el camino". (p. 43)

\subsection{Elevar la inversión en la educación y la equidad}

La expansión de la educación y la creación de verdaderas oportunidades para asegurar de forma sostenida dicho logro social requieren recursos de todo tipo. Se señala que para ampliar el acceso a los niveles medio y superior el costo promedio per cápita de la educación secundaria es 30 \% superior al costo de la educación primaria; costo que debe multiplicarse varias veces en el caso de la educación superior.

Trazarse la meta de mejorar la calidad de la educación en todos los niveles resulta muy difícil e igual de costosa, pues exige educadores mejor formados y remunerados, mejores edificaciones, menos estudiantes por docente, mejor equipamiento en las escuelas, laboratorios, computadoras y bibliotecas. Por un lado, de acuerdo con Schwartzman 2001):

Los países de la región invierten cerca del $12 \%$ de sus ingresos per cápita en educación, comparado con cerca del $19 \%$ de los países miembros de la OCDE. Un aumento del orden del $50 \%$ en el gasto per cápita, pondría a los países de la región a la par con los países de más altos niveles de inversión del mundo. Sin embargo, dados los ingresos sustancialmente más bajo de nuestros países, esto todavía no sería suficiente. Incluso en el mejor escenario posible, los países tendrán que elegir entre modestos mejoramientos de la educación primaria y secundaria y la expansión de la educación superior. (p. 12)

Por otro lado, según Rivas (2015):

Al valorar la inversión por alumno, que está altamente correlacionada con el peso del PBI. En América Latina el promedio de inversión anual por alumno del nivel secundario rondaba los 2.000 dólares mientras que en los países de Europa Occidental casi alcanzaba los 12.000 dólares con paridad cambiaria. Es indudable que esa diferencia de inversión por alumno tiene efectos diferenciales en la calidad educativa. (p. 194)

Diseñar políticas educativas coherentes con las demandas sociales y garantizar los recursos financieros necesarios para asegurar el desarrollo de manera sostenida son necesidades en los países de la región. Es necesario garantizar la infraestructura, incluida la tecnológica, y asegurar la remuneración debida del talento y la competencia profesional de 
los docentes de todos los niveles educativos. Elevar la inversión es necesario en todos los países, pero es tarea de cada cual definir hacia donde dirige dicha inversión.

\subsection{Políticas para el empleo de las TIC y los fundamentos pedagógicos necesarios}

El acceso y disponibilidad de la infraestructura no es suficiente. Estos tienen que estar interrelacionados con aspectos tales como el empleo sistemático, el desarrollo de capacidades para dar sustentabilidad de largo plazo; esto implica la formación de docentes, la capacitación de los administradores y la participación de los padres, entre otras, así como el desarrollo de contenidos educativos digitales de calidad que los docentes y estudiantes utilicen durante el proceso de enseñanza y aprendizaje (Sunkel, 2011).

La formación de competencias digitales es cada vez más importante en el ámbito educativo como una necesidad para la inclusión en la sociedad del conocimiento: las TICs no son solo un potente recurso para el aprendizaje, son herramientas cada vez más relevantes para la vida. El potencial de las TICs no se refiere sólo a la alfabetización digital, ellas pueden ser utilizadas para promover competencias modernas y mejorar el desempeño educativo de los estudiantes en términos generales. En los países de la región [el autor se refiere a Latinoamérica], el acceso a estas nuevas tecnologías en el hogar está fuertemente condicionado por el nivel socioeconómico de las familias, por lo que el sistema escolar ha sido la principal herramienta para reducir esta brecha tecnológica; aunque -ciertamente- ésta continúa siendo muy relevante en la mayoría de los países. Además de continuar equipando con TICs a las escuelas donde se educan quienes no pertenecen a los sectores privilegiados, el desafío futuro es cómo lograr que -más allá del uso recreativo- los estudiantes latinoamericanos les den un uso con potencial educativo; esto supone capacitar mejor a los docentes para incorporar a sus prácticas de enseñanza estas nuevas tecnologías. (Orealc/Unesco), 2013, p. 12)

Se requiere desarrollar un modelo pedagógico que permita abordar las políticas TIC de forma integrada y bajo los fundamentos de las ciencias de la educación. De esa manera podrá ser posible encarar los diferentes desafíos para cumplir con las grandes expectativas de las metas educativas y la política trazada. Las TIC vienen a ser un medio que dinamiza el aprendizaje y crea nuevas oportunidades educativas y, de manera especial, borra las 
fronteras del acto educativo en los límites de la escuela, pues favorece la adquisición autónoma de aprendizajes para toda la vida y en todos los espacios; para esto, la escuela y los maestros deben prepararse para lograrlo.

\subsection{Formación y perfeccionamiento continuo del docente}

El principal patrimonio de una nación y de una cultura es su capital humano. Si se entiende el desafío de esta manera, queda claro que la educación de los hombres y mujeres que guiarán nuestras naciones en términos de tiempo relativamente corto no puede estar en manos descuidadas y desmotivadas; tampoco pudieran estar guiados por personas inexpertas, poco o mal preparadas. La tecnología por sí sola es solo esto: un recurso importante, pero que no es capaz de formar a la personalidad de un niño o un adolescente. La formación humana, la educación de los sentimientos y las convicciones requieren de la acción de docentes competentes.

El pilar fundamental de la calidad educativa son las capacidades profesionales docentes: si los alumnos no se encuentran en sus aulas con docentes capaces de generar mayores oportunidades de aprendizaje, genuino mejoramiento de la calidad educativa no se producirá. Aunque la información disponible es parcial, todo indica que la situación predominante de la docencia en la región no se ajusta a las características de una profesión de alto estatus: los salarios y las condiciones laborales son deficientes, la formación inicial es de baja calidad, y las oportunidades de desarrollo profesional son limitadas. En consecuencia, el desafío de las políticas docentes es monumental: configurar una carrera profesional docente capaz de atraer a jóvenes talentosos a la docencia, formar adecuadamente a los candidatos, retener en las aulas (especialmente en aquellos sectores más desaventajados) a los profesores competentes, y hacer del desarrollo profesional una necesidad y una exigencia. Dado el carácter sistémico de todos estos procesos, es difícil avanzar en uno sin hacerlo en los demás. (Orealc/Unesco), 2013, p. 11)

La formación docente hoy requiere identificar referentes teórico-metodológicos claros, que emanen, ante todo, de nuestra cultura y que estén además a la altura de la ciencia y la tecnología en el mundo (Bruns y Luke, 2014). El modelo pedagógico que se tome debe ser consecuente con nuestra realidad y con sus demandas de formación. Son básicos, además de los saberes necesarios propios de su ciencia y disciplina particular, los contenidos de las 
ciencias de la educación, donde el aprendizaje en tecnología y para la tecnología resulta vital para un buen desempeño profesional en la contemporaneidad.

El rol del profesor (...) sigue siendo crucial, si bien ha cambiado su carácter. Actualmente, la misión del educador es cada vez menos la simple transmisión de conocimiento y más la de un mediador entre los estudiantes y la riqueza de información que los bombardea. La Internet, en la medida que se hace más eficiente y accesible, puede ser otro paso en esta dirección, ya que permite la comunicación entre el productor del contenido y el profesor, así como entre los propios profesores, y les brinda una oportunidad de buscar diferentes contenidos. (Schwartzman, 2001, p. 40)

La escuela y el maestro han de equiparse, no solo en instalaciones, mobiliario, recursos y tecnología, sino en saberes esenciales para lograr la formación de hombres y mujeres capaces de aprender por sí mismos, enriquecer su cultura y desarrollar una ética de paz y cuidado de la naturaleza en donde se vive, como primera noción de patria chica y autoctonía.

\section{Conclusiones}

El estudio de la educación, en su manifestación actual en la región latinoamericana, se presenta como un complejo objeto para su abordaje de manera objetiva y equilibrada. Se requiere tomar en cuenta, de forma compleja, la influencia de factores económicos, especialmente la pobreza y la exclusión social, unida a aspectos de carácter histórico, cultural, demográfico, así como la inversión en el sector y las políticas públicas relacionadas con este; es necesario valorar también la presencia coherente de las TIC en la escuela, y la manifestación que adquieren la formación y superación permanente del personal docente.

El estudio de la historia de la educación latinoamericana revela la larga lista de desencuentros e insatisfacciones acumuladas, que la adopción de disímiles políticas públicas y de gobierno no ha resuelto a través de siglos, propiciando una suerte de desfasaje en relación con los logros y metas situadas en occidente en su agenda educativa. La educación en el continente ha operado avances, ha evolucionado y ha sido campo de logros y grandezas, pero aún no ha sido posible despojarse de miserias y limitaciones que la pobreza y la desigualdad como pesados fardos imponen a millones de seres humanos en esta parte del mundo. 
Las reformas educativas no han inspirado transformaciones satisfactorias. El conservadurismo o tradicionalismo pedagógico, por una parte, y, por otra, el eclecticismo que ha animado a más de un proyecto transformador son factores que se asocian a los contenidos de los cursos, los aprendizajes logrados y las habilidades de los alumnos para aprender por sí a lo largo de toda la vida. Las transformaciones logradas, su calidad y ritmo de sucesión aún no resultan suficiente.

Es preciso reconceptualizar y revalorar la significación y el lugar de la escuela y, sobre todo, la profesión docente que, lejos de ceder espacio ante el avance abrumador de la tecnología, es cada vez más importante como punto de encuentro de estudiantes de todos los niveles educativos con su cultura, donde se aprende a convivir, a ser solidario y a vivir en paz. El desarrollo humano comprende la educación que propicia adquisiciones para aprender de forma creativa y crítica a través de toda la vida; esto solo será posible en una escuela inclusiva en todos los órdenes, una escuela y un docente actualizados en las ciencias y conocedores de la monumental cultura de la región. Una vez más, José Martí (1963) está presente y señala: "Injértese en nuestras repúblicas el mundo; pero el tronco ha de ser el de nuestras repúblicas" (Vol. 6, p. 18).

\section{Referencias}

Bruns, Bárbara y Luke, Javier. (2014). Como mejorar el aprendizaje en América Latina y el Caribe. Washington DC: Grupo del Banco Mundial.

Comisión Económica para América Latina y el Caribe (Cepal). (2014). Panorama social de América Latina. Santiago de Chile: Naciones Unidas/Cepal.

De la Luz, José. (1952). Escritos Educativos. La Habana: Universidad de la Habana.

Escribano, Elmys. (2015). La problemática de la relación de la cultura y la educación en América Latina. En Miriam Avellaneda y Alexandra Santos. Acercamiento a la enseñanza de la lengua y la formnación docente en América Latina (pp.19-32). Bogotá: Universidad Distrital Francisco José de Caldas.

Escribano, Elmys. (2016). El proyecto cultural liberador de José Martí para su tiempo y para el siglo XXI. Hallazgos, 13(25), 65-87. Recuperado de http://revistas.usta.edu.co/index.php/hallazgos/article/view/2557

García, José Félix. (2014). La dinámica de la pobreza en México. Una aproximación multidimensional. Investigación Operacional, 35(2), 141-147.

García, José Félix; Caamal, Ignacio y Priego, Oscar. (2016). Situación de pobreza y desigualdad en México. Una aproximación teórica. ATENAS, 1(33), 1-17. Recuperado de http://atenas.mes.edu.cu/index.php/atenas/article/view/182 
Guadarrama González, Pablo. (2008a). Humanismo práctico y desalienación en José Martí. En Pablo Guadarrama (Ed.), Pensamiento filosófico latinoamericano: humanismo vs alienación (Vol. I; pp. 360-362). Caracas: El Perro y la Rana.

Guadarrama González, Pablo. (2008b). Pensamiento filosófico latinoamericano: humanismo vs alienación. Caracas: El Perro y la Rana.

Instituto de Estadística de la Unesco. (2013). Uso de TIC en educación en América Latina y el Caribe. Análisis regional de la integración de las TIC en la educación y de la actitud digital (e-readiness). Montreal: Unesco.

Martí, José. (1963 ). Obras Completas (Vols. 7 y 19). La Habana: Editorial Nacional de Cuba.

Martins, Alejandra. (13 de mayo de 2015). 5 lecciones para América Latina del mayor ranking mundial de educación. BBC Mundo. Recuperado de http://www.bbc.com/mundo/noticias/2015/05/150513 educacion mapas am

Montiel, Edgar. (1995). Educación para la identidad. Educación, (85), 31-44.

Oficina Regional de Educación para América Latina y el Caribe (Orealc/Unesco). (2013). Situación educativa de América Latina y el Caribe. Santiago de Chile: Unesco.

Organización de Estados Iberoamericanos para la Educación, la Ciencia y la Cultura (OEI). (2014). Miradas sobre la educación en Iberoamérica. Avances en las metas educativas hacia 2021. Madrid: Organización de Estados Iberoamericanos para la Educación, la Ciencia y la Cultura (OEI).

Organización de las Naciones Unidas para la Educación, la Ciencia y la Cultura (Unesco). (2014). Primera entrega de resultados. TERCE. Tercer estudio regional comparativo y explicativo. Santiago de Chile: Unesco.

Organización de las Naciones Unidas para la Educación, la Ciencia y la Cultura (Unesco). (1974). Evolución Reciente de la Educación en América Latina. Santiago de Chile: Unesco.

Paz, Octavio. (1993). Los hijos del limo (4a. ed.). Barcelona: Barcelona: Seix Barral.

Pérez, Felipe. (2003). Pobreza y exclusión: Principales actores de la problemática educativa latinoamericana y caribeña. Cuadernos de Nuestra América, 16(31), 95-125.

Rivas, Axel. (2015). América Latina después de PISA. Lecciones aprendidas en siete países (2000-2015). Buenos Aires: Fundación Cippec.

Rumazo, Alfonso. (2008). Ideario de Simón Rodríguez. Caracas: Ministerio del Poder Popular para la Educación.

Sánchez, Gabriel. (11 de abril de 2015). ¿Por qué no mejora la educación en América Latina? BBC. Recuperado de http://www.bbc.com/mundo/noticias/2015/04/150409 economia educacion en americ a latina finde bd 
Schwartzman, Simon. (2001). El futuro de la Educación en América Latina y el Caribe. Santiago de Chile: Unesco.

Sunkel, Guillermo. (2011). TIC para la educación en América Latina: hacia una perspectiva integral. Recuperado de http://www.oei.es/noticias/spip.php?article9081

Tobón, Sergio. (2015). Necesidad de un nuevo modelo educativo para Latinoamérica. Revista Paradigma, 36(2), 5-6.

Tobón, Sergio, Gonzalez, Lourdes, Nambo, Juan Salvador y Vazquez, José Manuel. (2015). La socioformación: un estudio conceptual. Paradigma, 36(1), 7-29.

Torres, Carlos Alberto. (2001). Grandezas y miserias de la educación latinoamericana del siglo veinte. En Consejo Latinoamericano de Ciencias Sociales (Ed.), Paulo Freire y la agenda de la educación latinoamericana en el siglo XXI (pp. 23-52). Buenos Aires: CLACSO, Consejo Latinoamericano de Ciencias Sociales. Recuperado de http://bibliotecavirtual.clacso.org.ar/clacso/gt/20101010031230/2torres.pdf

Weinberg, Gregorio. (1981). Modelos educativos en el desarrollo histórico de América Latina. Buenos Aires: Unesco.

Zea, Leopoldo. (1976). El pensamiento latinoamericano. Barcelona: Ariel. 